\title{
Occupational therapy consensus recommendations for functional neurological disorder
}

\author{
Clare Nicholson (1) , ${ }^{1}$ Mark J Edwards, ${ }^{2}$ Alan J Carson, ${ }^{3}$ Paula Gardiner, ${ }_{1}^{4}$ \\ Dawn Golder, ${ }_{1}^{5}$ Kate Hayward, ${ }_{1}^{1}$ Susan Humblestone, ${ }_{1}^{6}$ Helen Jinadu, ${ }^{7}$ Carrie Lumsden, ${ }_{1}^{8}$ \\ Julie MacLean, ${ }^{9}$ Lynne Main, ${ }^{10}$ Lindsey Macgregor, ${ }^{11}$ Glenn Nielsen, ${ }^{2}$ Louise Oakley, ${ }^{12}$ \\ Jason Price, ${ }^{13}$ Jessica Ranford, ${ }^{9}$ Jasbir Ranu, ${ }^{1}$ Ed Sum, ${ }^{14}$ Jon Stone ${ }^{3}{ }^{3}$
}

\begin{abstract}
- Additional material is published online only. To view please visit the journal online (http://dx.doi.org/10.1136/ jnnp-2019-322281).
\end{abstract}

For numbered affiliations see end of article.

Correspondence to Mrs Clare Nicholson, Therapy Services, University College London Hospitals NHS

Foundation Trust National Hospital for Neurology and Neurosurgery, London WC1E 6BT, UK; clare.nicholson6@ nhs.net

Received 23 October 2019 Revised 12 March 2020 Accepted 1 April 2020 Published Online First 30 July 2020

Check for updates

(C) Author(s) (or their employer(s)) 2020. No commercial re-use. See rights and permissions. Published by BMJ.

To cite: Nicholson $\mathrm{C}$ Edwards MJ, Carson AJ, et al. J Neurol Neurosurg Psychiatry 2020:91:1037-1045.

\section{ABSTRACT \\ Background People with functional neurological disorder (FND) are commonly seen by occupational therapists; however, there are limited descriptions in the literature about the type of interventions that are likely to be helpful. This document aims to address this issue by providing consensus recommendations for occupational therapy assessment and intervention.}

Methods The recommendations were developed in four stages. Stage 1: an invitation was sent to occupational therapists with expertise in FND in different countries to complete two surveys exploring their opinions regarding best practice for assessment and interventions for FND. Stage 2: a face-to-face meeting of multidisciplinary clinical experts in FND discussed and debated the data from stage 1, aiming to achieve consensus on each issue. Stage 3: recommendations based on the meeting were drafted. Stage 4: successive drafts of recommendations were circulated among the multidisciplinary group until consensus was achieved.

Results We recommend that occupational therapy treatment for FND is based on a biopsychosocial aetiological framework. Education, rehabilitation within functional activity and the use of taught selfmanagement strategies are central to occupational therapy intervention for FND. Several aspects of occupational therapy for FND are distinct from therapy for other neurological conditions. Examples to illustrate the recommendations are included within this document. Conclusions Occupational therapists have an integral role in the multidisciplinary management of people with FND. This document forms a starting point for research aiming to develop evidence-based occupational therapy interventions for people with FND.

\section{INTRODUCTION}

Occupational therapists (OTs) assist people with physical and mental health difficulties across the lifespan to enable participation in daily activities. OTs are dually trained in physical and mental health rehabilitation. This skill set combined with a focus on function rather than impairment makes OTs ideally suited to help people with functional neurological disorder (FND).

FND is characterised by symptoms of altered voluntary motor or sensory function with clinical findings providing evidence of incompatibility between the symptoms and recognised neurological or medical conditions. ${ }^{1}$ Symptoms are diverse and can include weakness, movement disorders (tremor, jerks and dystonia), sensory symptoms, cognitive deficits and seizure-like events (commonly known as dissociative seizures or non-epileptic seizures). Fatigue and persistent pain are also commonly experienced as part of the disorder. Symptoms can present acutely and resolve quickly or can be long lasting. Regardless of duration, those affected frequently experience high levels of distress, disability, unemployment, social care utilisation and reduced quality of life. ${ }^{2}$ The stigma associated with FND contributes to the burden of the diagnosis. ${ }^{3}$

OT is generally recognised as an integral part of multidisciplinary rehabilitation for people with FND. As a therapy, it has face validity for FND; however, there is little published evidence to support its efficacy, and there are few published descriptions of interventions to guide practice. ${ }^{45}$ Given that FND differs in a number of important ways from other neurological conditions, typical OT neurorehabilitation strategies may not be directly translatable to people with FND and a more specific approach may be required.

The current evidence base for OT in FND rehabilitation is limited to several studies of multidisciplinary rehabilitation, ${ }^{6-9}$ including one with a randomised design. ${ }^{10}$ The interventions delivered by OTs in these studies are described only briefly; they include: retraining normal movement within function, graded reintroduction to daily activities, anxiety management and the reestablishment of structure and routine. Outcomes from these studies are promising, reporting improvement in scales of physical function and quality of life, immediately after treatment and at follow-up periods of 12-25 months. ${ }^{7-9}$ High levels of patient acceptability have been shown in at least one study that identified that OT compared favourably with other treatments. ${ }^{9}$ Evidence from randomised control trials is needed to demonstrate effectiveness of the specific rehabilitation interventions described in these studies.

In summary, OT is recognised as part of multidisciplinary intervention for FND; however, there is a limited evidence base, and the role of OT within the MDT is not well defined. With this paper, we aim to develop a broad set of consensus recommendations to guide OT practice for people with FND across the range of clinical settings (hospital, rehabilitation ward and community) and time following symptom onset (acute to chronic). These recommendations come from our experience working with people with FND aged 16 years and over; however, recommendations may have transferability to adolescent and 
paediatric populations. This work aims to complement existing clinical recommendations for $\mathrm{FND}^{11} 12$ and to form a starting point from which to develop evidence-based interventions.

\section{METHODS}

\section{Consensus process}

Data collection and analysis was based on a Delphi method, with four key stages.

Stage 1: in 2017/2018, 12 OTs from different countries with extensive experience in FND were invited to complete two online surveys exploring assessment and interventions for FND. The surveys were developed, data collated and summarised (by $\mathrm{CN})$. Elaboration and clarification were sought through a series of follow-up emails.

Stage 2: the expert OT group, together with experts in FND from other clinical disciplines were invited to take part in a face-to-face meeting in Edinburgh, UK, in September 2018. The collated data from stage 1 was discussed and debated.

Stage 3: recommendations based on the meeting were drafted.

Stage 4: a series of drafts were sent to the multidisciplinary group for feedback. Participants either endorsed each section of the draft or provided comments and suggestions. This process continued until a consensus was reached.

\section{Participants}

\section{Occupational therapists}

The group represented OTs from three nations: England $(n=7)$, Scotland $(n=3)$ and the USA $(n=2)$ who work with people with FND in a variety of clinical settings: acute neurological inpatients, neurological outpatients, neurological community rehabilitation, neuropsychiatry outpatients, neuropsychiatry inpatients, community mental health and accident and emergency. Participants had between 10 and 23 years (mean=17; $\mathrm{SD}=14$ ) of postgraduate experience.

\section{Multidisciplinary clinician group}

There were representatives from neuropsychiatry $(n=1)$, neurology $(n=2)$, neurophysiotherapy $(n=2)$ and neuropsychology $(n=1)$. There was also service user (patient and public) representation $(n=2)$.

All participants attended the meeting, except the OTs from the USA (who participated via Skype) and the neuropsychologist.

\section{Conceptualisation of FND/aetiological model}

The group's consensus on the definition and conceptual understanding of the diagnosis of FND was as follows: FND is a diagnosis that exists at the interface between neurology and psychiatry and is a genuine cause of disability due to motor and non-motor symptoms. Within the patient population, there is considerable heterogeneity in terms of symptom presentation, disability and psychiatric comorbidity. The aetiology is best understood within a biopsychosocial framework, considering predisposing, precipitating and perpetuating factors, each of which can include biological, psychological and/or social events (see table 1 below).

The motor symptoms of FND can be considered, at one level, as abnormal patterns of movement, that are 'driven' by involuntary self-focused attention. Thus, when the person's attention is redirected away from their body (distraction), abnormal movement reduces or disappears. Non-motor symptoms, including sensory changes, are also likely to be similarly affected by unconscious, self-focused attention.

People with FND commonly experience other health problems; this can include psychiatric comorbidity, coexisting neurological disease and other functional symptoms. Common coexisting symptoms such as chronic pain and fatigue are often important determinants of disability and quality of life. ${ }^{13} 14$

\section{Role and rationale for OT for FND}

The term 'occupational' in OT refers to any activity that has meaning and importance to an individual, based on what they need to do, want to do or are expected to do within societal and cultural norms. ${ }^{15}$

Common reasons to refer to occupational therapy:

- Disability affecting participation in daily activities (eg, personal care, domestic activities, childcare, community or leisure activities).

- Determining care needs for people with disability.

- Difficulty accessing home, education, work or community environments.

- Vocational rehabilitation and support to sustain paid employment, education and voluntary roles.

- Pain, fatigue, mental health and cognitive difficulties which impede activity engagement.

\section{When and how to refer to OT}

OTs can be involved from symptom onset to support in the community. It is preferable that a physician makes and explains the diagnosis to the patient prior to referring for treatment. ${ }^{16} 17$ We suggest sharing clinical consultation letters with the treating therapy team and the patient. A referral letter should include information such as the diagnosis, assessment findings, results from medical investigations, follow-up plans, other professionals involved and reason for referral.

Table 1 Potential predisposing, precipitating and perpetuating factors for FND (adapted from Stone ${ }^{50}$ )

\begin{tabular}{|c|c|c|c|}
\hline Factors & Biological & Psychological & Social \\
\hline Predisposing vulnerabilities & $\begin{array}{l}\text { Illness and disease. } \\
\text { History of previous functional symptoms. }\end{array}$ & $\begin{array}{l}\text { Personality traits. } \\
\text { Poor attachment/coping style. } \\
\text { Emotional disorder. }\end{array}$ & $\begin{array}{l}\text { Adverse life events or stressors. } \\
\text { Childhood neglect. } \\
\text { Difficulties in interpersonal relationships. } \\
\text { Symptom modelling. } \\
\text { Financial difficulties/deprivation. }\end{array}$ \\
\hline Precipitating mechanisms & $\begin{array}{l}\text { Physical injury or state (eg, drug side effect). } \\
\text { Abnormal physiological event (eg, hyperventilation } \\
\text { and sleep paralysis). }\end{array}$ & $\begin{array}{l}\text { Panic attack. } \\
\text { Perception of life event as traumatic/negative. }\end{array}$ & Adverse life events or stressors. \\
\hline Perpetuating factors & $\begin{array}{l}\text { Plasticity in sensory and motor pathways leading to } \\
\text { abnormal movement patterns. } \\
\text { Deconditioning. } \\
\text { Fatigue. } \\
\text { Chronic pain. }\end{array}$ & $\begin{array}{l}\text { Illness beliefs (person and significant others). } \\
\text { Feeling disbelieved. } \\
\text { Maladaptive behaviours. } \\
\text { Co-morbidities including anxiety and depression. }\end{array}$ & $\begin{array}{l}\text { Diagnostic uncertainty (eg, ongoing medical } \\
\text { investigations). } \\
\text { Reliance on care and benefits. } \\
\text { Compensation claims. } \\
\text { Ongoing social stressors (eg, relationship difficulties, } \\
\text { financial hardship and loss of roles). }\end{array}$ \\
\hline
\end{tabular}


Different factors may influence the suitability for treatment. The group suggest that OT intervention is more likely to be successful if the person with FND:

- Has some degree of understanding and agreement with the diagnosis.

- Has agreed to the referral.

- Can identify rehabilitation goals or areas of need and are motivated to make changes.

- Has an understanding that the initial focus of OT is to improve function and therefore is unlikely to include provision of aids and adaptations.

It is not uncommon for people to lack confidence in the diagnosis of FND. Through providing education about the diagnosis at a level that is accessible to the patient, OT can help to turn around low diagnostic confidence. In instances where there is still doubt, we would suggest that OT can still be of benefit by focusing on the impact of symptoms on function rather than addressing symptoms at a diagnostic or impairment level. However, where there is strong disagreement about the diagnosis, therapy is much less likely to be helpful.

\section{Assessment and outcome measurement}

OT assessment for people with FND is usually undertaken over a number of sessions. Taking time to listen to the person and explore the impact of their symptoms is key to developing a positive therapeutic relationship, as well as understanding their rehabilitation needs. The following structure may be useful to guide an initial assessment:

1. Ask the person about when and how their symptoms started.

2. Create a list of current symptoms. For each symptom, ask about exacerbating and easing factors, variability, severity and the impact on function.

3. Clarify the person's understanding of their diagnosis and whether they are in agreement with it.

4. Ask about other health problems and medical history (including psychological health).

5. Take a detailed social history, exploring usual roles, responsibilities and meaningful activities.

6. Gain a picture of their 24-hour routine. This is helpful to uncover symptom perpetuating factors such as poor sleep hygiene, boom and bust activity patterns, lack of occupation and structure.

7. Determine if they have care needs, and if so, are these needs being met and by whom?

8. Ask about access to the home, education or work environment and the use of environmental adaptations and aids.

9. If they are in work or education, ask about the impact of their symptoms. If unemployed or retired, it may be appropriate to ask about receipt of benefits/insurance.

10. Undertake an observation of daily activities (eg, personal care, meal preparation and functional transfers) to establish the impact of symptoms on activity engagement. During observation, note distractibility and variability of symptoms so that these can be discussed in a supportive way. ${ }^{18}$

Identify the person's goals for treatment and their expectations of OT. It is important to come to a shared understanding of what treatment can be offered; a verbal agreement at the start of intervention can prevent potential difficulties when concluding treatment. We suggest discussing that OT intervention will be directed by the goals or problems that the person has identified and that independent practise outside of sessions will be required. If, after some discussion, the person is unable
Table 2 Useful occupational therapy specific assessment tools for FND

The Canadian Occupational Evidence-based outcome measure that captures a person's selfPerformance Measure perception of performance and satisfaction with performance in daily activities.

Has been widely adopted in occupational therapy research. Used for both paediatric and adult populations. ${ }^{51}$

Occupational Semistructured interview design based on the $\mathrm{MOHO}$; captures Circumstances Assessment information regarding occupational engagement from 11 domains

Interview and Rating Scale including habits, roles, personal causation, values, skills, physical environment, social environment and readiness for change. ${ }^{52}$

The Worker Role Interview Semistructured interview format addressing factors that may impact on a person's ability to maintain or return to work during or after a period of illness or injury. ${ }^{53}$

The Model of Human Assesses the impact of a person's volition, habituation, skills and Occupation Screening Tool environment on their occupational functioning. ${ }^{54}$

The Occupational Self- Based on the MOHO

Assessment Captures a person's perceptions of their own occupational competence and occupations of importance.

Therapist observations are also considered when scoring. ${ }^{55}$

Adolescent and Adult Evaluates behavioural responses to everyday sensory experiences. Sensory Profile Used for ages older than 11 years. ${ }^{41}$

The Assessment of Motor Clinician rated tool. Allows therapists to simultaneously observe and Process Skills and evaluate a person's ability to perform activities of daily living (domestic and personal) and the quality of their motor and process skills. ${ }^{56}$

FND, functional neurological disorder; $\mathrm{MOHO}$, Model of Human Occupation.

to identify goals or areas of need, then it may be appropriate to discuss whether OT intervention is right for them at this time.

Unique to OT is the use of occupation-based conceptual models of practice that guide intervention. The two models of practice most commonly used by the consensus group are the Model of Human Occupation and the Canadian Model of Occupational Performance and Engagement. ${ }^{19} 20$ Other models may be helpful, but these two were favoured owing to their links with well-established tools that are used by the consensus group to guide assessment and intervention with FND (see table 2). Although none have been specifically validated in an FND population, these tools can be also be used to measure the outcomes of intervention.

There is an absence of clinically useful, validated outcome measures specific to FND. Assessing change in FND is challenging due to the heterogeneity of presentations, variability of symptom severity and multiple interacting comorbidities. Outcome measures should therefore address multiple domains including physical function and disability, quality of life, mental health, healthcare utilisation and perception of symptom severity. We direct readers to a recent systematic review of outcome measurement in FND and recommendations by Nicholson et al and Pick et al. ${ }^{21} 22$

\section{OT treatment}

OT aims to help people overcome the effects of disability through practical support to improve performance and satisfaction in activities of daily living. The consensus group identified that core OT interventions for FND include physical rehabilitation through guided activity practise, practical management of pain and fatigue, support to address mental health problems and support to reduce dependency and optimise independence.

The group suggests that supporting self-management of symptoms is particularly important in FND and should be considered through all aspects of OT. The person is supported to take ownership of their rehabilitation and develop an internal locus of control, rather than placing control in the hands of clinicians and family members. This is achieved through understanding the diagnosis, understanding the rationale for interventions and 
graded goal setting. Interactive workbooks or a therapy journal can be a useful tool to support self-management.

\section{Goal setting}

Goal setting is generally considered an important part of OT intervention. ${ }^{23}$ Goal setting with people with FND can differ from methods used in typical neurorehabilitation settings, for example, after stroke, where therapists may be able to relatively accurately predict recovery and help set realistic goals. With FND, it can be helpful to consider that recovery often follows a pattern of symptom remission and exacerbation. For this reason, most of the consensus group reported that they take a more flexible approach to goal setting; goals are set by the person with FND, in their own words and may not necessarily be time dependent.

\section{Education}

Education to improve understanding about symptoms is widely considered to be an important part of FND treatment. ${ }^{2425}$ Helping people with FND to understand the diagnosis can improve their confidence that it is correct and builds the foundations for rehabilitation and self-management. It is important that OTs are aware that there is often stigma attached to the diagnosis of FND. Patients report that interactions with healthcare professionals have often left them feeling misunderstood and abandoned by the healthcare system. ${ }^{3}$ Clinicians should therefore be mindful to impart information with empathy and sensitivity, emphasising the genuine nature of symptoms. Education should include how activities and occupations, combined with taught strategies, form part of symptom management. It is important to pitch education at the right level, recognising the person's attitude, knowledge and skills in order to change, support and enhance occupational performance. ${ }^{26}$ See Box 1 for points that can be included in an explanation of FND and the role of OT.

\section{Vocational rehabilitation}

OTs have a key role in supporting people with FND to manage their condition in the context of work and/or study. ${ }^{27}$ We suggest that vocational rehabilitation principles for neurological rehabilitation can be applied to people with FND. ${ }^{28}$ This includes helping employers and educators to understand that they have genuine symptoms that may vary in severity, with possible periods of remission and exacerbation. OTs can work jointly with occupational health departments to identify and advocate for reasonable adjustments to improve work performance and symptom management. This may include role modifications, reduced hours, regular rest breaks and flexible working options. Another important aspect of vocational rehabilitation is to develop a graded return to work/study following a period of sick leave. ${ }^{29}$

Sustaining paid employment is likely to be important in maintaining good health; however, in some cases, it may not be possible for the person to continue despite reasonable adjustments. ${ }^{30}$ In these cases, the consensus group suggests that OTs can have a role in supporting people with FND to positively withdraw from their current role and seek alternative roles (paid or unpaid).

\section{Aids and adaptations}

The use of aids and equipment is generally thought to be unhelpful to the rehabilitation process in FND, potentially preventing future improvement by interrupting normal automatic movement patterns and causing maladaptive ways of functioning.
Box 1 Ingredients of education about functional neurological disorder (FND)

1. Acknowledge that FND is a real, common and disabling condition that causes neurological symptoms that are outside the person's control.

2. The symptoms of FND are caused by a potentially reversible miscommunication between the brain and the body.

3. Discuss that FND is diagnosed by a neurologist using 'positive clinical signs' (eg, Hoover's sign, distractibility, entrainment of tremor and variability in symptoms during function) and is not a diagnosis of exclusion. It can be diagnosed alongside other neurological conditions. ${ }^{18}$

4. Help the person understand the relevance of triggering events if they report them. ${ }^{11}$

5. Discuss how self-directed attention can make symptoms worse and conversely redirecting attention (distraction) can temporarily reduce symptoms.

6. Provide education on how the body's stress response can influence functioning of the nervous system and therefore FND.

7. Discuss how symptom focus and chronic health conditions can exacerbate or cause cognitive difficulties, fatigue, anxiety, low mood and pain.

8. Discuss the role of OT in treating FND.

9. Discuss the importance of a 24-hour approach to therapy; that is, implementing rehabilitation strategies throughout the daily routine as part of self-management.

10. Signpost to sources of information including: www. neurosymptoms.org and to patient-led organisations, for example, FND Hope International, FND Hope UK, FND Hope US, FND Hope Canada, FND Action (UK), FND Dimensions (UK) and FND Australia Support Services. The book Overcoming Functional Neurological Symptoms: Five Areas Approach ${ }^{24}$ can be a useful resource in helping people understand symptoms and management strategies, especially in those that recognise anxiety and/or mood instability to be part of their problem.

It is important to recognise that aids and equipment can cause new secondary problems such as joint pain (eg, shoulder pain from walking with crutches) and deconditioning of muscles. ${ }^{11}$ The group endorsed these concerns but recognised that there are times when providing adaptive equipment is appropriate and/or necessary. Each case should be considered individually. It can be helpful to consider the person's journey, differentiating the acute phase where improvement is more likely, from situations where people have treatment resistant symptoms. In the acute phase, it is advisable to avoid aids and environmental adaptations. If aids are necessary, for example, to enable a safe discharge from hospital, equipment should be: (1) considered as a short-term solution; (2) issued with a minimalist approach; and (3) a plan to progress from its use. It is important to assess the person with new equipment and teach them how to use it correctly to minimise maladaptive movement patterns. Follow-up appointments should be available to monitor equipment use and support plans to progress towards independence.

For people who have completed rehabilitation but experience ongoing disability, it is reasonable and appropriate to consider aids and environmental adaptations. Equipment should maximise safety, increase independence, improve community access 


\begin{tabular}{|c|c|}
\hline Symptom & Intervention strategy \\
\hline Functional tremor & $\begin{array}{l}\text { Superimpose alternative, voluntary, 'rhythms' on top of the existing tremor and gradually slowing all movement to a complete rest. } \\
\text { Unilateral tremor: use the unaffected limb to dictate a new rhythm (eg, tapping/opening and closing the hand), that is entrain the tremor to stillness. } \\
\text { Music can be introduced to dictate a rhythm to follow. } \\
\text { Assist the person to relax the muscles in the limb to prevent cocontraction. } \\
\text { Try to control a tremor with the person at rest, before moving on to activity. } \\
\text { Use of gross rather than fine movements (which take more concentration), for example, handwriting retraining; using a marker and large piece of paper or } \\
\text { white board with big lettering or patterns/shapes rather than trying to focus on 'normal' handwriting. } \\
\text { Discourage cocontraction or tensing of muscles as a method to suppress a tremor, as this is unlikely to be a helpful long-term strategy. }\end{array}$ \\
\hline Functional jerks & $\begin{array}{l}\text { Addressing unhelpful prejerk cognitions and movement (eg, signs of anxiety, frustration or effort, such as breath-holding). } \\
\text { General relaxation techniques;: diaphragmatic breathing or progressive muscular relaxation. } \\
\text { Sensory grounding; a strategy that can be used to bring oneself into the present moment }{ }^{40} \text { (eg, noticing details in the environment (sounds, sights and } \\
\text { smells), feeling a textured item, cognitive distractors such as counting backwards and singing). } \\
\text { Encourage learning of 'slow' movement activities such as yoga or tai chi as a way of regaining movement control and redirecting attention away from the } \\
\text { symptom. }\end{array}$ \\
\hline Dystonia & $\begin{array}{l}\text { Encouraging optimal postural alignment at rest and within function, considering a 24-hour management approach. } \\
\text { Encourage even distribution of weight in sitting, transfers, standing and walking to normalise movement patterns and muscle activity. } \\
\text { Grade activity to increase the time that the affected limb is used (using normal movement techniques) within functional activities. } \\
\text { Avoid postures that promote prolonged positioning of joints at the end of range (eg, full hip, knee or ankle flexion while sitting). } \\
\text { Discourage nursing of the affected limb but demonstrate and promote therapeutic resting postures and limb use. } \\
\text { Strategies that reduce muscle overactivity, pain and fatigue, for example, muscle relaxation strategies, supporting the affected limb when at rest, using } \\
\text { pillows or furniture to take the weight of a limb when sitting or lying down. } \\
\text { Address associated problems of pain and hypersensitivity. }\end{array}$ \\
\hline Functional limb weakness & $\begin{array}{l}\text { Engage the person in tasks that promote normal movement, good alignment and even weight-bearing. Task examples may include: transfers, sit to stand, } \\
\text { standing, perch sitting in personal care or kitchen tasks, using the hand to stabilise objects (so as to avoid learnt non-use) and placing the hand on the } \\
\text { kitchen bench while standing to prepare food (rather than letting it hang by the side). } \\
\text { Bilateral functional lower limb weakness; joint sessions with PT colleagues to complete tasks using the upper limbs while standing with the aid of a } \\
\text { standing frame. }\end{array}$ \\
\hline
\end{tabular}

With all symptom types, employing anxiety management and distraction techniques when undertaking a task can be helpful. Video recording interventions (with consent) can be useful to play back to the person to identify changes in symptoms (eg, in tremor amplitude or extinction). It can demonstrate changeability, highlight successes (and build confidence) and act as a reference point for replication of strategies outside of therapy.

and have an overall positive impact on quality of life. See the online extended version of this document for further details.

\section{Splinting}

As with adaptive aids, splinting may prevent restoration of normal movement and function. Potential problems with splinting are: (1) increasing attention and focus to the area, thereby exacerbating symptoms; (2) increasing accessory muscle use, (3) use of compensatory movement strategies, (4) immobilisation leading to muscle deconditioning; (5) learnt non-use and (6) increased pain. Serial casting for fixed functional dystonia has been associated with worsening symptoms and the onset of complex regional pain syndrome. ${ }^{31} 32$

We suggest trying strategies that encourage normal movement patterns and resting postures before considering splinting. It is possible that removable splints may have an overall positive effect in some cases, but benefits should be balanced against potential harm. If a splint is issued, the person should be monitored regularly and feel empowered to discontinue use in the event of adverse side effects such as pain and skin break down.

\section{Symptom-specific treatment suggestions}

\section{Functional motor symptoms}

Functional motor symptoms include tremor, weakness, dystonia, gait disorder and jerks. Movement strategies that redirect attention away from the body can help to reduce motor symptoms and normalise movement (see table 3 for examples). OTs can help people identify and practise normal movement strategies and integrate them into daily activities.

\section{Functional visual impairment}

Functional visual loss may be experienced as a persistent absence of vision, intermittent loss of sight or reduced visual acuity. ${ }^{33}$ While the person may experience a lack of vision, it is usually possible to observe actions that demonstrate that they are making use of visual information in an automatic (or subconscious) way. For example, they may avoid obstacles while walking or pick up an object without guidance. While there is little published information regarding treatment, there was experience within the consensus group of helping people with functional visual loss, including a description of treatment for two patients who had a positive outcome. ${ }^{34}$ This approach included: (1) helping the person understand the diagnosis and (2) sensitively communicating to the person and positively reinforcing events when they are likely to have used visual information (although without awareness). OT intervention should aim to prevent activity avoidance and dependence on others. OTs also have a role in assisting to minimise secondary problems associated with light hypersensitivity, agoraphobia and unhelpful behaviours such as keeping the eyes closed.

\section{Functional cognitive impairment}

Cognitive symptoms are common in FND either as a primary problem or as part of a combination of symptoms. Several factors can contribute to cognitive problems including pain, fatigue, anxiety, low mood, poor sleep, sensory hypersensitivity, symptom focus and medication side effects. ${ }^{35}$ Before advising practical cognitive strategies, it is helpful to discuss the link between potential contributing factors and cognitive function. The aim is to understand, and where appropriate normalise, these experiences and reframe the symptom as part of FND rather than an additional health problem. Discussions should help the person to understand that their cognitive skills are likely intact (unless there is underlying comorbidity), but multiple factors are competing for their cognitive resources.

Key to managing cognitive symptoms is to address the contributing factors (fatigue, pain, anxiety and poor sleep), either as part of OT or to encourage the person to seek help from their general practitioner (eg, to consider reducing sedating medications). Other helpful strategies include encouraging structure 
and routine; writing out a daily plan to prevent activity and cognitive overload; taking time out for relaxation (to minimise stress); and normal use of (but not dependence on) calendars and alarm functions in mobile phones. OTs can also support the person and significant others to gradually reduce practical assistance (if applicable) and provide opportunity for positive risk taking within function. Finally, like other functional symptoms, overly attending to the problem (ie, trying to remember) is unhelpful; most people can relate to the experience of remembering a forgotten name once they have stopped trying to think about it.

\section{Dissociative (non-epileptic) seizures (DSs)}

DSs are a specific presentation of FND characterised by temporary episodes of impaired awareness. The episodes may resemble epilepsy or syncope, but they are not associated with abnormal electroencephalography changes. DSs are a common reason for frequent attendance to accident and emergency. ${ }^{36}$ Acute hospital admissions can be highly distressing and are usually unnecessary for DS. The main evidence-based treatment for DS involves psychological therapy, delivered by specially trained clinicians. ${ }^{37}$ Here we provide ways in which core OT skills can be used to help people with problems associated with DS. See the online extended version of this document for further information.

A good place to start treatment is to ask the person how they wish to be supported if they have a DS during a therapy session. They may have devised a plan with other clinicians that you can follow. If not, this could be an initial focus of OT intervention. A DS plan can help put clinicians at ease and make the person feel safer, which may prevent escalation and reduce duration of the episode. ${ }^{38}$ Important elements are to help the person to a safe space where they are unlikely to injure themselves. Let them know they are safe but avoid constant reassurance and physical contact or restraint. Advising others to behave as they would if someone is having a panic attack can be helpful. People can sometimes hear and understand what you say during a DS, even if they are unable to respond.

Ask about triggers and warning signs of an imminent episode. It is common for people to report having no memory of events prior to and during a DS; however after some discussion, many start to recognise patterns. If a warning sign is experienced prior to a DS, taught strategies can be used to try and avert an event at this point. ${ }^{39}$ A group of strategies that can be helpful are Sensory Grounding Techniques, which aim to keep people present in the moment and focus attention to prevent dissociation. ${ }^{40}$ Some examples include noticing the detail in the environment (eg, colours, textures and sounds); cognitive distractions (eg, word games and counting backwards); and sensory-based distractors (eg, flicking a rubber band on the wrist and feeling a textured item).

\section{Common problems associated with FND Hypersensitivity}

Many people with FND report hypersensitivity to touch, light, sound and movement. Specific terms have been used to describe these problems, including sensory modulation difficulties, sensory defensiveness and sensory over-responsiveness. ${ }^{41} 42$ These symptoms are not unique to FND; in fact, they are also common in people with other diagnoses, such as migraine, chronic pain and fatigue. It is important to address hypersensitivity as it can become a maintaining factor, resulting in avoidant behaviours, limiting participation in functional activities and exacerbating other symptoms and disability.
The experience of hypersensitivity can influence the pace and intensity at which treatment can be delivered and should therefore be carefully assessed and considered as part of triage and treatment planning. UK group members outlined that sensory hypersensitivity (for people with FND) tends to be addressed within function by encouraging graded exposure to various sensations experienced within the persons day-to-day routine. The UK group also endorsed gradual minimisation of compensatory techniques such as use of sunglasses when indoors for photophobia or the use of headphones or ear plugs for auditory sensitivity or the more content-specific misophonia, as these are thought to perpetuate and exacerbate hypersensitivity. In some settings in the USA, treatment and assessment of sensory modulation difficulties are based on the Jane Ayers Theory of Sensory Integration. ${ }^{43}$ The Adolescent/Adult Sensory Profile assessment tool can be used to direct the development of a personalised sensory exposure regimen. This regimen may combine compensatory strategies to inhibit and grade exposure to sensory sensitivities alongside sensory-based activities to better regulate emotional responses, improve cognitive functioning and increase functional participation.

Hypersensitivity of the skin or allodynia is a common secondary consequence of 'fixed' functional dystonia (as are other trophic changes associated with complex regional pain syndrome). ${ }^{31}$ Unchecked, hypersensitivity can lead to problems associated with learnt non-use (eg, further pain and hypersensitivity, muscle atrophy and discomfort associated with overuse of the unaffected side). OTs can facilitate graded use of the affected limb while gradually reducing protective postures. For instance, encouraging arm swing when walking, weight-bearing in sitting or standing, bilateral upper limb use in activity. Graded exposure to different sensory experiences may help to normalise sensation, for example, wearing clothing on the affected limb, applying moisturiser and washing up in warm water.

\section{Anxiety}

Although not universal, anxiety is common in FND and may act as a symptom precipitating or perpetuating factor. Some people may experience the physiological and somatic consequences of anxiety (eg, racing heart rate and tight chest) without recognising the experience emotionally. This has been described as panic without panic or alexithymia. ${ }^{44}$ OTs can help to address anxiety through education by describing the physiological process of anxiety and its physical impact on the body. The concept of a fight or flight response may be useful for patients who do not identify as feeling anxious. OTs can help people identify how anxiety interacts with FND symptoms to impede involvement in daily activities and implement anxiety management strategies. This can include breathing techniques, progressive muscle relaxation, grounding strategies, visualisation, distraction, thought reframing, mindfulness, integrating activities of enjoyment, regular cardiovascular exercise and supporting the person to undertake an anxiety provoking task in graded steps.

\section{Fatigue and pain management}

People with FND often experience pain and/or fatigue. These can be disabling symptoms and should therefore be considered as part of any intervention. Evidence for the management of pain and fatigue in other long-term conditions currently exist and can be adapted for use in FND. ${ }^{45} 46$ 
Psychological trauma

A systematic review found that serious adverse life events are more common in people with FND compared with the general population. ${ }^{47}$ Of note, childhood neglect was found to be a greater risk factor than physical or sexual abuse. Importantly, adverse life events were not universal, and even if present, may be relevant to some but not all as also evidenced by a recent case controlled study exploring predisposing risk factors for functional limb weakness. ${ }^{48}$ For some people, addressing the impact of adverse events (eg, in post-traumatic stress disorder) may be a necessary part of treatment. For others, a more symptom focused intervention may be appropriate. The group felt that addressing the sequelae of psychological trauma is not a generic OT role. Some OTs have sufficient experience and qualifications to manage difficulties that arise from serious mental health problems, but this usually is the result of additional training and accreditation. However, people sometimes disclose information about traumatic events during OT intervention. It is therefore important that therapists have the skills to listen and contain the person's distress. Using reflexivity to acknowledge one's own limitations within the context of your professional role is imperative. There are some OTs who may feel more capable in managing trauma due to their skill set and practice setting (eg, mental health or neuropsychiatry settings). Less experienced clinicians should be encouraged to seek supervision and support. Knowing who to refer on to if a person discloses trauma (and consents to onward referral) is important and clear lines of how to do this should be established.

\section{Risk management}

People with FND often disengage from usual activities due to a perceived risk to their personal safety (eg, fear of having a DS on leaving the house). It is an OTs' role to help manage risk and increase independence by enabling people to engage in activities through a process of supported positive risk taking. This concept is explored in a recently published guidance document for OTs in the UK, 'Embracing risk; enabling choice'. ${ }^{49}$ The identified principles can be applied to working with people with FND and the group endorses its guidance. Further information is available in the extended online document.

\section{Disability management}

Care

OTs can provide advice on the need for carers to assist with activities of daily living and the extent of care required. If carers are needed, care that provides opportunity for the person to be actively involved in tasks (facilitatory) rather than having a task completed for them (passive care) should be encouraged.

\section{Benefits}

People with FND are entitled to health and social care benefits/insurance but many find the systems involved difficult to navigate. It may therefore be necessary and appropriate for OTs to support applications for benefits and insurance claims. This may involve liaising with agencies, supporting the completion of paperwork and advocating on the person's behalf regarding the potential benefit of undergoing specialist rehabilitation (eg, a letter outlining treatment options and the evidence base). A simple 'to whom it may concern' letter, describing a person's diagnosis, articulating the finer details of the condition (eg, variability of symptoms), impact on function and their support needs can be a valuable tool to help people negotiate with relevant agencies. When patients are on the cusp of eligibility for benefits and undergoing treatment, an open discussion about whether they wish to defer application until they see the outcome of treatment may be appropriate.

\section{Housing}

Rehousing to an adapted property should be avoided when symptoms are presenting acutely and/or access to rehabilitation is available and wanted. Support with rehousing may be appropriate if a patient has chronic symptoms that have not responded to a rehabilitation approach. A person's housing situation may also be a trigger or maintaining factor to their symptoms or lack of occupational independence and therefore if no immediate changes can be affected, then rehousing may be considered. In such instances, OTs may have a role in advocating for rehousing on behalf of the person.

\section{Relapse prevention/staying well plan}

During rehabilitation and the journey to recovery, it is common for people with FND to experience periods of symptom exacerbation. OTs should help people to prepare to manage these episodes with a relapse prevention, management or staying well plan. This essential part of treatment is a written summary that can be completed collaboratively at the conclusion of treatment. A plan should consider the following questions:

1. What have you learnt about your condition?

2. What makes your symptoms worse?/What might trigger setbacks?

3. What are the most helpful management strategies that you have learnt?

4. What were the unhelpful coping strategies that were making it difficult for you to improve?

5. What can you do if you notice that your symptoms and function are getting worse?

6. What are your goals for the next 3, 6, 9 and 12 months? (using a graded goal setting approach).

\section{Concluding treatment}

It can be difficult to bring an intervention period to an end if the person continues to experience debilitating symptoms. Commencing the intervention with a treatment agreement (as described above) may help with this process. It is important to maintain the therapeutic relationship, promote confidence and self-efficacy by fostering the person's ability to continue with their self-management plan. In this way, discharge should be framed not as concluding treatment but rather the point in which the person should independently continue to implement their self-management strategies.

There will be some people who do not gain benefit from rehabilitation and who remain symptomatic. If the person has had adequate access to specialist intervention for FND then a focus on disability management may be appropriate. However, given the fluctuating nature of FND, it is important to consider that gains may still be possible in the future. Re-engaging in rehabilitation at a later date may be advantageous for some. Booking in a follow-up appointment to review progress, troubleshoot issues and reset goals as the system allows is advised. Peer support organisations can be an important adjunct to treatment, especially for people who continue to experience symptoms.

See Box 2 below for a summary of the intervention principles for OT and FND suggested in this document. 
Box 2 Summary of intervention principles for occupational therapy and functional neurological disorder (FND)

- Be empathic and recognise that FND is a real and disabling condition.

- Take the time to listen to the person's story and build therapeutic rapport.

- Agree treatment parameters at the start of your intervention.

- Introduce the concept of self-management at initial assessment.

- Provide education about the diagnosis and the person's symptoms. Involve significant others in education and treatment.

- Use variability of symptoms on examination and in day-today life positively in treatment.

- Recognise and sensitively challenge unhelpful thoughts, beliefs and behaviours.

- Integrate specific treatment techniques into function and show the person how to carry these over independently.

- Focus on activity-based (functional) rather than impairmentbased goals and interventions.

- Be open and consistent in your verbal and written communications with the patient and other healthcare professionals.

- Where possible avoid use of compensatory aids and techniques in the acute phase or when undergoing active rehabilitation.

- Avoid use of splints or devices that immobilise joints.

- Complete a relapse prevention and ongoing self-management plan as part of treatment.

\section{LIMITATIONS}

Due to a lack of evidence for specific OT interventions, the recommendations provided in this paper are based on expert opinion. Where possible, we have drawn on evidence from multidisciplinary interventional trials and other therapies. These recommendations are aimed at adult patients. Interventions for children and adolescents with FND are likely to be similar to those described here but may differ in some areas. Treatment advice for younger people is an important direction for future publications. Our international authorship increases the generalisability of the recommendations; however, the information may be less transferable in nations not represented.

\section{CONCLUSIONS/SUMMARY}

FND is a complex condition that has far reaching consequences on physical and psychological health, occupational participation and quality of life. Current best practice is for multidisciplinary rehabilitation, of which OT is a part. However, there are few resources available to help guide OT assessment and intervention. Here we provide recommendations for OT assessment and intervention for people with FND, considering their journey from acute presentation to support in the community. The information within this document highlights the important role of OT in helping people with FND and may have relevance for other professional groups. Future research should seek to test the individual components of these recommendations and explore the potential for cost benefit. Additional material is published online only (online supplementary figure 1).

\section{Author affiliations}

'Department of Therapy Services, University College London Hospitals NHS Foundation Trust National Hospital for Neurology and Neurosurgery, London, UK ${ }^{2}$ Neurosciences Research Centre, University of London St George's Molecular and Clinical Sciences Research Institute, London, UK

${ }^{3}$ Centre for Clinical Brain Sciences, University of Edinburgh, Western General Hospital, Edinburgh, UK

${ }^{4}$ Centre for Clinical Brain Services, University of Edinburgh, NHS Lothian, Edinburgh, UK

${ }^{5}$ FND Hope UK, London, UK

${ }^{6}$ Department of Neuropsychiatry, University College London Hospitals NHS Foundation Trust, National Hospital for Neurology \& Neurosurgery, London, United Kingdom

${ }^{7}$ The Lishman Unit, South London and Maudsley NHS Foundation Trust, London, United Kingdom

${ }^{8}$ Community Rehabilitation and Brain Injury Service, West Lothian Community Health and Care Partnership, Livingston, West Lothian, United Kingdom

${ }^{9}$ Occupational Therapy Department, Massachusetts General Hospital, Boston, Massachusetts, United States

${ }^{10}$ Neurological rehabilitation out-patient services, Astley Ainslie Hospital, NHS Lothian, Edinburgh, Scotland, United Kingdom

${ }^{11}$ Falkirk Community Mental Health Service for Adults, NHS Forth Valley, Falkirk, Scotland, United Kingdom

${ }^{12}$ National Centre for Mental Health, Birmingham and Solihull Mental Health NHS Foundation Trust, Birmingham, United Kingdom

${ }^{13}$ Department of Neuropsychology, James Cook University Hospital, South Tees Hospitals NHS Foundation Trust, Middlesbrough, United Kingdom

${ }^{14}$ Integrated Community Neurology Team, East Coast Community Healthcare CIC, Lowestoft, United Kingdom

\section{Twitter Alan J Carson @alancarson15 and Jon Stone @jonstoneneuro}

Acknowledgements AJC acknowledges submitting paid testimony in court actions on a range of neuropsychiatric topics, receiving payment as editor of Journal of Neurology Neurosurgery and Psychiatry, royalties from BMJ press and Elsevier for books and he is the unpaid treasurer of the Functional Neurological Disorder Society. JS receives royalties from UpToDate Inc and acknowledges paid testimony as an expert witness in court in relation to functional neurological disorders. He is supported by NHS Scotland Career Fellowship and runs the free website https://www.neurosymptoms.org/ for patients with functional neurological disorders.

Contributors $\mathrm{CN}$ developed the assessment and intervention surveys, collated and summarised the survey data. The occupational therapists in the group: $\mathrm{CN}, \mathrm{KH}, \mathrm{SH}$, HJ, CL, JM, LyM, LiM, LO, JeR, JaR and ES completed the online surveys and follow up discussions. All authors attended the consensus meeting and agreed on content to be included in the manuscript. CN, GN, KH, JS, MJE and AC prepared the first draft of the manuscript. All authors were given the opportunity to review and revise the manuscript.

Funding MJE has received research funding from the Medical Research Council, National Institute for Health Research and the Guarantors of Brain; honoraria from Merz Pharma, TEVA and UCB; and publishing royalties from Oxford University

Press. GN receives research funding from the National Institute for Health Research.

Competing interests None declared.

Patient consent for publication Not required.

Provenance and peer review Not commissioned; externally peer reviewed.

\section{ORCID iDs}

Clare Nicholson http://orcid.org/0000-0002-8047-6820

Jon Stone http://orcid.org/0000-0001-9829-8092

\section{REFERENCES}

1 Association APTF on D-I. Diagnostic and statistical manual of mental disorders:DSMIV 1994.

2 Carson A, Stone J, Hibberd C, et al. Disability, distress and unemployment in neurology outpatients with symptoms 'unexplained by organic disease'. J Neurol Neurosurg Psychiatry 2011;82:810-3.

3 Nielsen G, Buszewicz M, Edwards MJ, et al. A qualitative study of the experiences and perceptions of patients with functional motor disorder. Disabil Rehabil 2018;0:1-6.

4 Gardiner P, MacGregor L, Carson A, et al. Occupational therapy for functional neurological disorders: a scoping review and agenda for research. CNS Spectr 2018;23:205-12

5 Ranford J, Perez DL, MacLean J. Additional occupational therapy considerations for functional neurological disorders: a potential role for sensory processing. CNS Spectr 2018;23:194-5. 
6 McCormack R, Moriarty J, Mellers JD, et al. Specialist inpatient treatment for severe motor conversion disorder: a retrospective comparative study. J Neurol Neurosurg Psychiatry 2014;85:895-900.

7 Demartini B, Batla A, Petrochilos P, et al. Multidisciplinary treatment for functional neurological symptoms: a prospective study. J Neurol 2014;261:2370-7.

8 Czarnecki K, Thompson JM, Seime R, et al. Functional movement disorders: successful treatment with a physical therapy rehabilitation protocol. Parkinsonism Relat Disord 2012;18:247-51.

9 Saifee TA, Kassavetis P, Pareés I, et al. Inpatient treatment of functional motor symptoms: a long-term follow-up study. J Neurol 2012;259:1958-63.

10 Jordbru AA, Smedstad LM, Klungsøyr O, et al. Psychogenic gait disorder: a randomized controlled trial of physical rehabilitation with one-year follow-up. J Rehabil Med 2014:46:181-7.

11 Nielsen G, Stone J, Matthews A, et al. Physiotherapy for functional motor disorders: a consensus recommendation. J Neurol Neurosurg Psychiatry 2015;86:1113-9.

12 Carson A, Lehn A, Ludwig L, et al. Explaining functional disorders in the neurology clinic: a photo story. Pract Neurol 2016;16:56-61.

13 Gelauff JM, Kingma EM, Kalkman JS, et al. Fatigue, not self-rated motor symptom severity, affects quality of life in functional motor disorders. J Neurol 2018;265:1803-9.

14 Věchetová G, Slovák M, Kemlink D, et al. The impact of non-motor symptoms on the health-related quality of life in patients with functional movement disorders. J Psychosom Res 2018;115:32-7.

15 Therapists WF of 0, 2012. Available: http://www.wfot.org/about-occupational-therapy

16 Espay AJ, Aybek S, Carson A, et al. Current concepts in diagnosis and treatment of functional neurological disorders. JAMA Neurol 2018;75:1132-41.

17 Stone J. Functional neurological disorders: the neurological assessment as treatment. Neurophysiol Clin 2014:44:363-73.

18 Stone J, Edwards M. Trick or treat? showing patients with functional (psychogenic) motor symptoms their physical signs. Neurology 2012;79:282-4.

19 Kielhofner G. A model of human occupation. theory and application. Baltimore: Williams \& Wilkins, 1985

20 Polatajko HJ, CJ TEA. Enabling Occupation II: Advancing an Occupational Therapy Vision of Health, Well-being, \& Justice through Occupation. Ottawa: CAOT Publications ACE, 2007.

21 Nicholson TR, Carson A, Edwards MJ, et al. Outcome measures for functional neurological disorder: a review of the theoretical complexities. J Neuropsychiatry Clin Neurosci 2020;32:33-42.

22 Pick S, Anderson DG, Asadi-Pooya AA, Pooya AAA-, et al. Outcome measurement in functional neurological disorder: a systematic review and recommendations. J Neurol Neurosurg Psychiatry 2020;91:638-49.

23 Therapists RC. Goals of occupational therapy intervention. London, 2016

24 Williams C, Kent C, Smith S, et al. Overcoming functional neurological symptoms: a five areas approach. Hodder Arnold, 2011.

25 Nielsen G. Chapter 45 - physical treatment of functional neurologic disorders, 1st ED Elsevier B.V 2016.

26 Jain S, Kings J, Playford ED. Occupational therapy for people with progressive neurological disorders: unpacking the black box. British Journal of Occupational Therapy 2005;68:125-30.

27 College of Occupational Therapists College of Occupational Therapists. Occupational therapy evidence: vocational rehabilitation Factsheet. Available: https:/www.rcot.co. uk/sites/default/files/VR factsheet.pdf [Accessed 7 Jun 2019].

28 Tyerman A \& MM. Vocational assessment and rehabilitation after acquired brain injury:interagency guidelines. London: British Society of Rehabilitation Medicine / Royal College of Physicians, 2004.

29 Sweetland J, Howse E, Playford ED. A systematic review of research undertaken in vocational rehabilitation for people with multiple sclerosis. Disabil Rehabil 2012;34:2031-8.

30 Health D. W and P and D of improving lives: the work, health and disability green paper. London, 2016
31 Schrag A, Trimble M, Quinn N, et al. The syndrome of fixed dystonia: an evaluation of 103 patients. Brain 2004;127:2360-72.

32 Popkirov S, Hoeritzauer I, Colvin L, et al. Complex regional pain syndrome and functional neurological disorders - time for reconciliation. J Neurol Neurosurg Psychiatry 2019;90:608-14.

33 Bruce BB, Newman NJ. Functional visual loss. Neurol Clin 2010;28:789-802.

34 Stone J, Yeo JM CA. Lessons from successful treatment of functional visual loss using diagnostic transparency, occipital transcranial magnetic stimulation and hypnotherapy. Pract Neurol 2018;19:168-72.

35 Stone J, Pal S, Blackburn D, et al. Functional (psychogenic) cognitive disorders: a perspective from the neurology clinic. J Alzheimers Dis 2015;48 Suppl 1:S5-17.

36 Duncan R, Graham CD, Oto M, et al. Primary and secondary care attendance, anticonvulsant and antidepressant use and psychiatric contact 5-10 years after diagnosis in 188 patients with psychogenic non-epileptic seizures. J Neuro/ Neurosurg Psychiatry 2014;85:954-8.

37 Goldstein LH, Mellers JDC, Landau S, et al. Cognitive behavioural therapy vs standardised medical care for adults with dissociative non-epileptic seizures (codes): a multicentre randomised controlled trial protocol. BMC Neurol 2015;15:98.

38 Reuber M. Dissociative (non-epileptic) seizures: tackling common challenges after the diagnosis. Pract Neurol 2019;19:332-41.

39 LaFrance WC, Reuber M, Goldstein LH. Management of psychogenic nonepileptic seizures. Epilepsia 2013;54 Suppl 1:53-67.

40 Goldstein LH, Chalder T, Chigwedere C, et al. Cognitive-Behavioral therapy for psychogenic nonepileptic seizures: a pilot RCT. Neurology 2010;74:1986-94.

41 Brown C, Tollefson N, Dunn W, et al. The adult sensory profile: measuring patterns of sensory processing. Am J Occup Ther 2001;55:75-82.

42 C T. Sensory Modulation \& Environment: Essential Elements of Occupation. Pearson Australia Group, 2011.

43 A J. Occupational therapy for motor disorders resulting from impairment of the central nervous system. Rehabil Lit 1974;21:302-10.

44 Mellers JDC. The approach to patients with "non-epileptic seizures". Postgrad Med J 2005:81:498-504.

45 The British Pain Society. The British pain Society. guidelines for pain management programs for adults. London 2013.

46 Thomas S, Thomas PW, Kersten P, et al. A pragmatic parallel arm multi-centre randomised controlled trial to assess the effectiveness and cost-effectiveness of a group-based fatigue management programme (facets) for people with multiple sclerosis. J Neurol Neurosurg Psychiatry 2013;84:1092-9.

47 Ludwig L, Pasman JA, Nicholson T, et al. Stressful life events and maltreatment in conversion (functional neurological) disorder: systematic review and meta-analysis of case-control studies. Lancet Psychiatry 2018;5:307-20.

48 Stone J, Warlow C, Deary I, et al. Predisposing risk factors for functional limb weakness: a case-control study. J Neuropsychiatry Clin Neurosci. In Press 2020;32:1-8.

49 Therapists RC. Embracing risk; enabling choice-Guidance for occupational therapists. London, 2017.

50 Stone J. Function symptoms in neurology. Neurol Pract 2009:9:179-89.

51 Carswell A, McColl MA, Baptiste $\mathrm{S}$, et al. The Canadian occupational performance measure: a research and clinical literature review. Can J Occup Ther 2004;71:210-22.

52 Forsyth K, Deshpande S, Kielhofner G, et al. OCAIRS: the occupational circumstances assessment interview and rating scale, 4.0. Chicago: MOHO Clearinghouse, 2005.

53 Braveman B, Robson M, Velozo C, et al. Worker role interview. Chicago, WRI: MOHO Clearinghouse, 2005

54 Parkinson S, Forsyth K, Kielhofner G. Model of human occupation screening tool (MOHOST). Chicago: University of Illinois at Chicago, 2006.

55 Baron K, Kielhofner G, Lyenger A, et al. The occupational self assessment (version 2.2) model of human occupation. Chicago: Clearinghouse, 2006.

56 AG F. Assessment of motor and process skills. Three Star Press: Fort Collins Co, 2003. 